\title{
Corrigendum to "Applications of Dendrimers in Drug Delivery Agents, Diagnosis, Therapy, and Detection"
}

\author{
B. Noriega-Luna $\mathbb{D}^{1}{ }^{1}$ Luis A. Godínez, ${ }^{1}$ F. J. Rodríguez, ${ }^{1}$ A. Rodríguez $\mathbb{D}^{1}$ \\ G. Zaldívar-Lelo de Larrea, ${ }^{2}$ C. F. Sosa-Ferreyra, ${ }^{2}$ R. F. Mercado-Curiel ${ }^{\circ},{ }^{2} \mathrm{~J}_{\text {. Manríquez, }}{ }^{1}$ \\ and E. Bustos $\left.{ }^{1}\right)^{1}$ \\ ${ }^{1}$ Centro de Investigación y Desarrollo Tecnológico en Electroquímica S.C., Parque Tecnológico Querétaro s/n, Sanfandila, \\ Pedro Escobedo, Querétaro, QRO C.P. 76703, Mexico \\ ${ }^{2}$ Medicine Faculty, Universidad Autónoma de Querétaro, Fracc. Prados de la Capilla, Querétaro, QRO C.P. 76176, Mexico \\ Correspondence should be addressed to E. Bustos; ebustos@cideteq.mx
}

Received 16 October 2019; Accepted 22 October 2019; Published 23 January 2020

Copyright (C) 2020 B. Noriega-Luna et al. This is an open access article distributed under the Creative Commons Attribution License, which permits unrestricted use, distribution, and reproduction in any medium, provided the original work is properly cited.

The article titled "Applications of Dendrimers in Drug Delivery Agents, Diagnosis, Therapy and Detection: An Overview" [1], was found to contain a substantial amount of material, without citation, from previously published articles. This has been corrected in the revised version shown below:

(i) The complete article has been updated

(ii) Five references were added [2-5]

[28] V. Gajbhiye, V. K. Palanirajan, R. K. Tekade and N. K. Jain, "Dendrimers as therapeutic agents: a systematic review," Journal of Pharmacy and Pharmacology, vol. 61, pp 989-1003, 2009.

[39] J. M. Oliveira, A. J. Salgado, N. Sousa, J. F. Mano and R. L. Reis, "Dendrimers and derivatives as a potential therapeutic tool in regenerative medicine strategies - a review," Progress in Polymer Sciences, vol. 35, no. 9, pp. 1163-1194, 2010.

[102] D. Astruc, E. Boisselier and C. Ornelas, "Dendrimers Designed for Functions: From Physical, Photophysical, and Supramolecular Properties to Applications in Sensing, Catalysis, Molecular Electronics, Photonics, and Nanomedicine," Chemical Reviews, vol. 110, pp. 1857-1959, 2010.

[105] S. Svenson, "Dendrimers," In Kirk-Othmer Encyclopedia of Chemical Technology. Copyright John Wiley \& Sons, Inc., vol. 26, pp. 786-812, 2006.

[112] N. Joshi and M. Grinstaff, "Applications of dendrimers in tissue engineering," Current Topics in Medicinal Chemistry, vol. 8, no. 14, pp. 1225-1236, 2008.

\section{Abstract}

The application of dendrimers in biomedicine has recently attracted much attention worldwide. Dendrimers are interesting in the field of biomedical applications due to their unique characteristics. Among these properties, the hyperbranched, well-defined globular structure, multivalency, variable and tunable chemical composition, and high biological compatibility stand out. In particular, it is important to note that the tridimensional architecture of dendrimers allows the incorporation of biologically active agents to form the corresponding conjugates. In this context, this review focuses on the application of dendrimers in interesting and promising applications, such as the design and construction of drug delivery agents and chemically modified electrodes.

\section{Introduction}

Dendrimers, also known as cascade polymers, are tridimensional structures that were first reported by Gajbhiye et al. in 2009 [2]. These unimolecular micelles were also the basis for polypropylene imine (PPI) dendrimers, constructed by Oliveira et al. and Astruc et al. in 2010 [3-4]. In 2006, Svenson also reported a very interesting type of dendrimer prepared from a mixture of amines and amides, which are known as poly(amido amines) or PAMAM dendrimers [5]. Several dendrimers were reported in the 1980s and 1990s, and in general terms, these molecules are widely 
known as tree-, branch-, arborol- or cascade-shaped polymers.

The dendritic branching leads to semiglobular and globular configurations with a large number of terminal functional groups that define their reactivity and interaction with the molecular environment. The central core, on the other hand, could be a single atom or molecule with multiple branching peripheral groups from which the hyperbranching building blocks are sequentially connected. This region around the central dendrimer unit is characterized not only by empty spaces in which small organic and/or inorganic molecules can be located but also by chemical functionalities responsible for a variety of supramolecular interactions that can be exploited to form an interesting and potentially important array of weak complexes.

As previously mentioned, the particular structure and space configuration of dendrimers give rise to different physicochemical characteristics that can be used to define and exploit internal as well as external interactions with other organic and/or inorganic molecules. In fact, the branching structure that characterizes dendrimer molecules (with diameters $<10 \mathrm{~nm}$ ) resembles, from a generic point of view, not only the shape of some proteins and biomolecules (biomimics) but also their ability to interact with other organic molecules by encapsulation to increase their solubility, disperse active molecules, and enhance the administration and transport properties in a given medium, as well as by other different mechanisms, or to improve pharmaceutical activity to treat memory impairment, inflammatory diseases, microbial infections, and cancer.

In this review, we study the applications of dendrimers as protein mimics, delivery agents for drugs, and anticancer or antiviral therapeutics. In addition, we discuss the use of dendrimers in biomedical diagnostic applications such as modified electrodes.

\section{Dendrimers as Possible Globular Protein Mimics}

Considering the particular internal and external structure and morphology of dendrimers, these molecules can be used for biomimetic studies to better understand the function and dynamics of some biological systems. In this regard, PAMAM dendrimers have been used as molecular structure analogs of insulin (generation 3.0 with a diameter of $3 \mathrm{~nm}$ ), cytochrome C (generation 4.0 with a diameter of $4 \mathrm{~nm}$ ), and hemoglobin (generation 5.0 with a diameter of $5.5 \mathrm{~nm}$, with an ammonia functional group in the central core).

Although dendrimers are similar to some important proteins and, as has been pointed out, can be used to mimic these biomolecules and gain a better understanding of their structure-activity relationship, it is also true that there are important differences between proteins and dendrimers and that these must be taken into account so that a proper comparison can be made. As opposed to proteins whose structure (and function) usually obeys a weak balance of supramolecular interactions that are asymmetric in different directions, dendrimers are similar to unimolecular covalent micelles that are characterized by a large resistance to environmental changes such as alterations in temperature, light, and $\mathrm{pH}$. Therefore, the stability of the internal and external complexation suites is far greater than that in proteins (see, for example, Figure 1). The next sections show examples of mimetic applications of dendrimers.

2.1. Dendrimers Mimicking Proteins Involved in Angiogenesis. Dendrimers have been prepared and used to mimic the protein region involved in the angiogenesis inhibition of b-FGF in endothelial cells (Figure 2(a)) so that antitumor processes can be better understood. In vitro and in vivo studies (Figure 2(b)) have been carried out to prevent the growth and metastasis of tumors.

2.2. Biomimetic Regeneration of Hydroxyapatite. Dental enamel can be used to mineralize tissue in the human body, and different procedures are in place to form enamel-like crystals aimed at mimicking the biomineralization process of enamel. One study of the biomimetic regeneration of hydroxyapatite was performed by Chen et al. (2003-2004), in which an enamel crystal was capped with PAMAM dendrimers. Recently, Wu et al. (2013) synthesized a carboxyl-terminated poly(amido amine)- (PAMAM$\mathrm{COOH}-$ ) alendronate (ALN) conjugate (ALN-PAMAM$\mathrm{COOH}$ ) to mimic biomineralization (Figure 3). Another example is the work of Chen et al. (2013), who studied the effect of G3.0 PAMAM-COOH on crystal growth on etched enamel in a short time and same crystallographic orientation.

2.3. Collagen-Mimetic Dendrimers. Gajbhiye et al. (2009) reported the potential for the synthesis of artificial collagen in the context of avoiding disease transmission and allergic responses when natural tissue is employed. In this context, Kinberger et al. (2002) studied the synthesis of collagenmimetic dendrimers (PAMAM G1.0 core) with GlyPro-Nleu and Gly-Nleu-Pro sequences exhibiting thermal stability. Gajbhiye et al. (2009) and Kinberger et al. (2002) reported the synthesis of PAMAM collagen-mimetic molecules previously complexed with $\mathrm{Cu}^{2+}$ and $\mathrm{Ni}^{2+}$, obtaining extraordinary biological properties.

\section{Dendrimers as Drug Delivery Agents}

Due to their physicochemical structure, internal void regions, and external or peripheral surface functional groups, dendrimers have been widely studied as drug delivery agents. Furthermore, due to the sequential nature of the most common synthetic procedures, controllable structure, size, internal and terminal functional groups, permeability, hydrodynamic volume, molecular weight, molecular geometry, and variation in the internal and external charge can be designed and incorporated into the final dendrimer structure. With all these characteristics, dendrimers have been used to retain drugs, enzymes, antibodies, and other bioactive agents (Figure 4).

3.1. Dendrimers in Drug Administration. One interesting approach for the development of drug delivery dendrimers was a report on the use of PAMAM dendrimer generation 3.0 and 3.0-lauroyl to study their effect on the transport of 


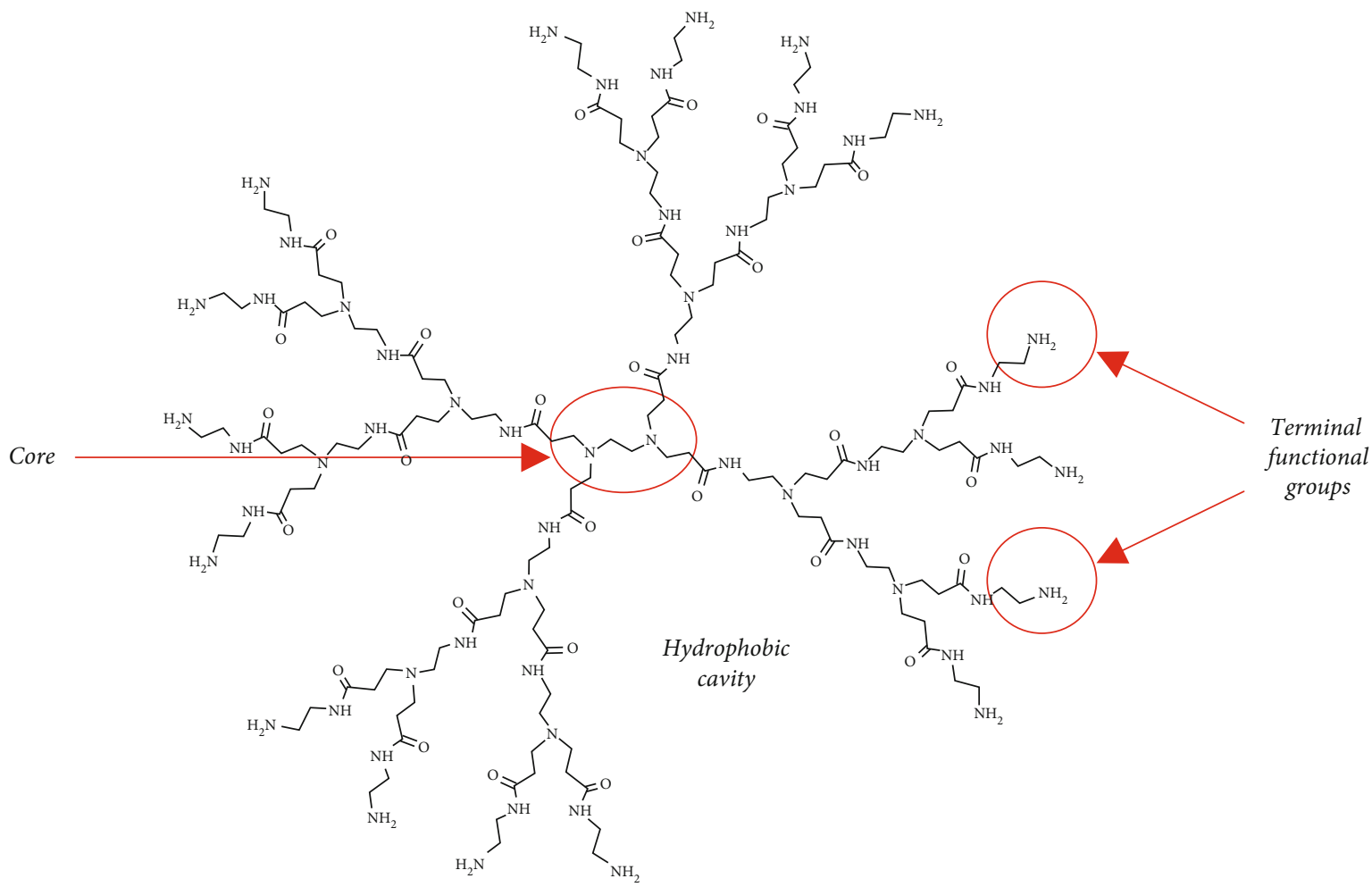

FIgURE 1: Representation of G2.0 PAMAM with 16 terminal amine groups.

propranolol to the adenocarcinoma cell line CaCo-2. This phenomenon involves endocytosis-mediated transepithelial transport (Figure 5) to increase drug solubility and bypass drug efflux transporters, improving the drug bioavailability.

PAMAM dendrimers have also been used as drug carriers of ketoprofen and camptothecin. The corresponding studies in vitro and in vivo showed that the dendrimer molecules were capable of increasing the solubility of the drug in water and eliminating the negative effect in the gastrointestinal tract. In the case of the PAMAM dendrimer-ketoprofen complex, a favorable effect was also found in terms of anti-inflammatory, antipyretic, and analgesic properties. On the other hand, in the case of the PAMAM dendrimer-camptothecin complex, promising results were found in anticancer therapy (including lung, prostate, breast, colon, stomach, bladder, and ovarian cancer as well as melanoma). In addition, PAMAM dendrimer generation 3.5-SN38 (7-ethyl-10hydroxy-camptothecin) without and with CaCo-2 monolayers or PAMAM dendrimer generation 3.5-glycine-SN38 has shown interesting properties in terms of stability in the gastric and intestinal region by oral administration, without cytotoxicity, targeting the treatment of colorectal hepatic metastasis.

\section{Dendrimers as Carriers or Scaffolds for Diagnosis and Therapy}

Dendrimers have a number of advantages for use as carriers or scaffolds for diagnosis and therapy. For example, the amphiphilic property and interior cavities of dendrimers can be used to encapsulate hydrophobic or hydrophilic drugs depending on the dendrimer components. In addition, the generation-size linear correlation of dendrimers implies that they can be used as suitably sized molecules for specific biomedical applications.

4.1. Dendrimers as Anticancer Drug Carriers. The most promising uses of dendrimers are as drug carriers due to their globular shape and their combined hydrophobic and hydrophilic character. In this way, properly positioned functional groups in the internal or peripherical zone can retain organic and inorganic compounds by means of dynamic supramolecular interactions, such as those found in DNA (for gene delivery), cisplatin (anticancer drug), and silver salts (antimicrobial activity against Staphylococcus aureus, Pseudomonas aeruginosa, and Escherichia coli bacteria).

4.2. Dendrimers as MRI Contrast Agents. Magnetic resonance imaging (MRI) is widely used to obtain tridimensional images in vivo without ionizing radiation. Using a gadolinium chelate contrast agent, superparamagnetic iron oxide particles and hepatobiliary contrast agents are administered before the scan. Among the main properties that are required for contrast agents, the need to diffuse rapidly from blood vessels into the interstitial space and to be efficiently excreted from the body without accumulation are two of the most important. In this regard, Gd(III)-2-(4-isothiocyanato-benzyl)-6-methyl-(DTPA)-PAMAM dendrimers have been found to be suitable for in vivo experiments with rats.

4.3. Dendrimers in Tissue Regeneration. Tissue regeneration technology consists of promoting the healing or construction 


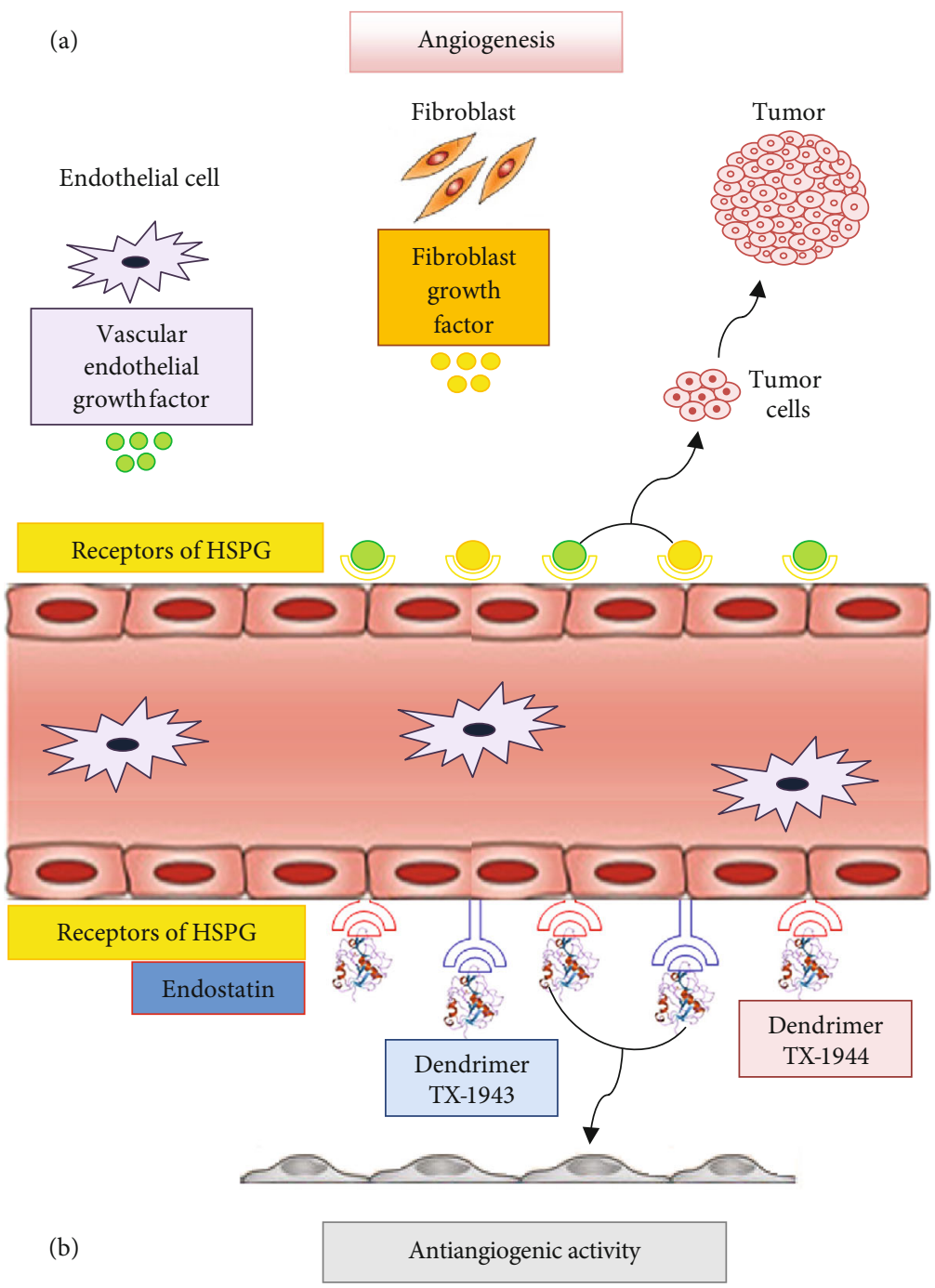

FIGURE 2: Schematic representation of (a) the process of angiogenesis and (b) the antiangiogenic activity of arginine with dendrimers TX-1944 and TX-1943

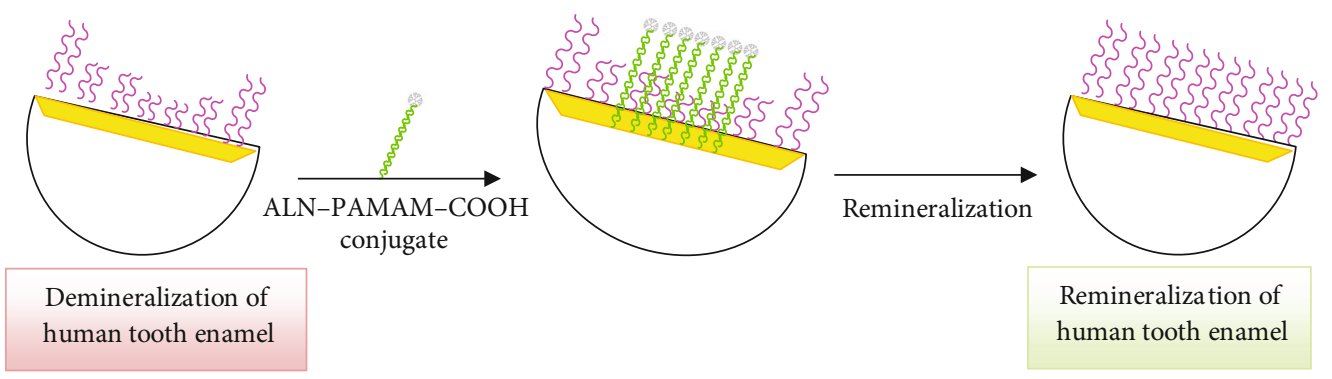

FIGURE 3: Schematic representation of the regeneration process of hydroxyapatite with alendronate (ALN) conjugated to carboxyl-terminated poly(amido amine) (PAMAM).

of living tissue for organs, which can in turn be used in transplant procedures [6]. To do so, biocompatible matrices are necessary to support or encapsulate cellular material so that the living cells can have access to nutrients and eliminate metabolic waste. Among the natural polymers that have been explored for tissue regeneration, collagen, fibrin, hyaluronic acid, chondroitin sulfate, alginate, dextran, and chitosan stand out as the most popular materials due to their biocompatible nature and their availability. On the other hand, in the case of synthetic polymers, both linear and three-dimensional polymers are studied. To this end, linear polymeric chains such as poly(lactic acid) (PLA), poly(glycolic acid) (PGA), poly(caprolactone) (PCL), and poly(ethylene glycol) (PEG) have been explored. 

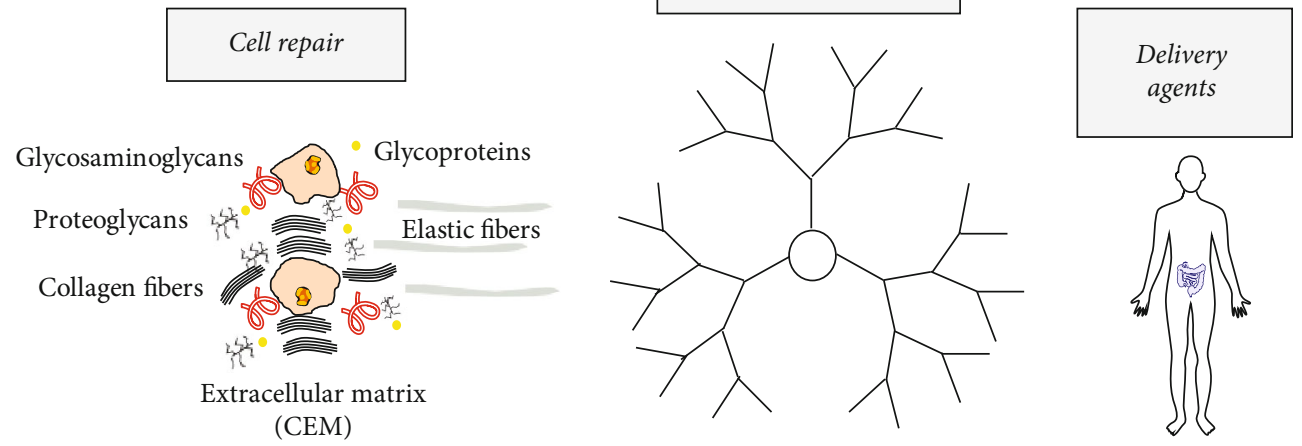

FIGURE 4: Schematic representation of the use of dendrimers in different medical applications: cell repair, diagnosis, and drug delivery.

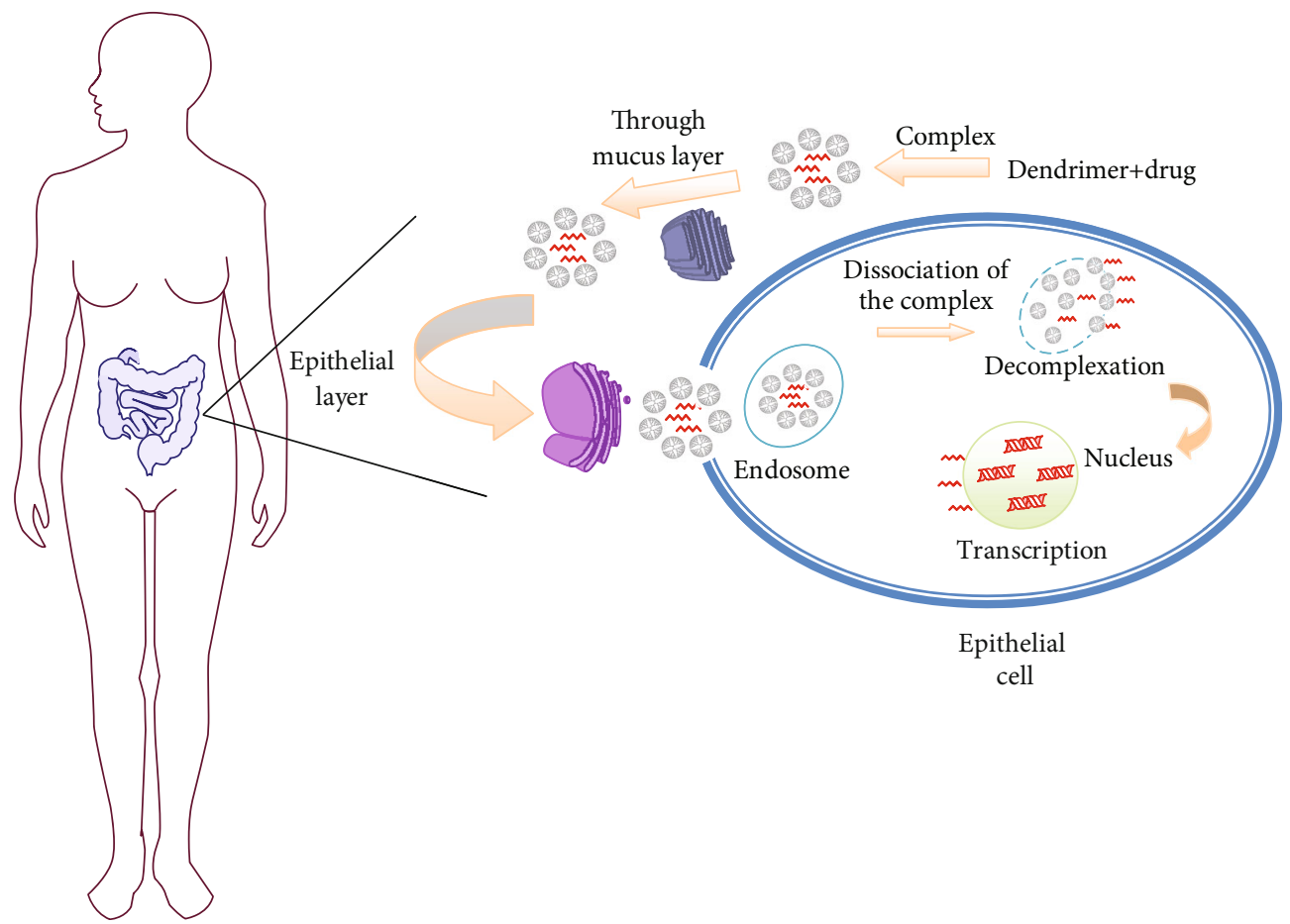

FIGURE 5: Schematic representation of the transepithelial transport mechanism of the dendrimer-drug complex.

4.3.1. Dendrimers in Cell Repair. For porous polymeric extracellular matrices (ECMs) that can accommodate and supply a proper environment to repair tissues, dendrimers have been used in in vivo studies. PAMAM dendrimer generation 1.0 in particular has been employed for the regeneration of porcine tissue. Another application of dendrimers in this field corresponds to in vitro bone tissue reconstruction using poly(caprolactone)-hydroxyapatite films or the poly(caprolactone)- (PCL-) poly(L-lysine) dendritic core.
4.4. Dendrimers in Vaccine Development. Vaccination undoubtedly has been one of the most important and successful strategies implemented worldwide for the control of infectious diseases. In the field of vaccine design, dendrimers can be used as model molecules to host immunostimulators and/or antigens.

4.4.1. Dendrimers as Antigens. In the context of the ongoing efforts to develop an acquired immunodeficiency syndrome 


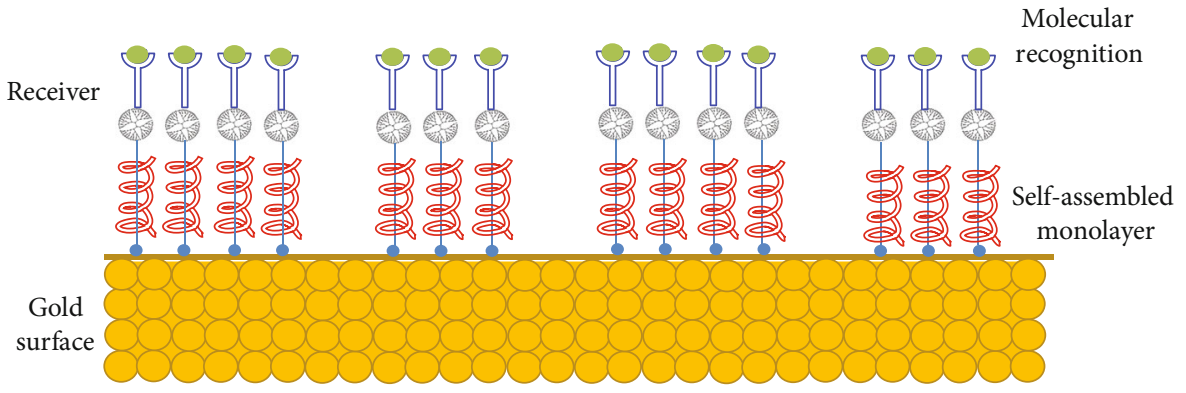

FIGURE 6: Schematic representation of an electrode modified with a self-assembly monolayer on a gold surface.

(AIDS) vaccine, there are some interesting reports involving dendrimers. Among these, studies involving a major antigenic peptide (MAP) with glycoprotein gp120 of the human immunodeficiency virus (HIV) sequence conjugated to a lysine dendrimer or a peptide-succinyl-maltose-prolinepoly(lysine) dendrimer can be readily found. Therefore, the inclusion of dendrimers in these vaccines should increase their water solubility and decrease cell toxicity and antigenicity. Other antigen-related studies involving dendrimer molecules report the use of polyacrylate dendritic polymers to eliminate infection with Streptococcus pyogenes, commonly known as group A Streptococcus (GAS), in rheumatic fever and heart disease (RHD), antigen peptide of $P$. yoelii by polyclonal antibodies, MAP-containing Plasmodium falciparum T- and B-cell stimulatory peptides, and MAP-sensitized dendritic cells and P815 cell lysis by pRL1a-specific B-24 CTL.

\section{Dendrimers in Modified Electrodes}

In clinical chemistry, the food industry, and different sectors of the environmental field, there is an increasing need for efficient, cost-competitive, and reliable electrochemical sensors. In this regard, surface modification of conductor electrodes serving as supporting substrates is by far the most common approach; in particular, the layer-by-layer (LBL) assembly method is one of the most important for the design and construction of bioelectrochemical detectors using organic or inorganic molecules such as proteins, DNA, viruses, dendrimers, and nanoparticles with unique properties. In the particular case of dendrimers, their unique supramolecular properties have been used in LBL electrochemical sensors to detect and quantify molecules of biological importance. Using specific physicochemical properties such as encapsulation capacity, molecular control, hydrophobicity/hydrophilicity biocompatibility, controlled diffusion, and electron transfer kinetic effects, dendrimers are currently positioned as an important class of surface modification compounds for the design and construction of electrochemical sensors.

5.1. Electrochemical Detection in Medical Diagnosis. Detection and quantification in medical diagnosis can be made using optical, electrochemical, or piezoelectric transduction techniques. The electrochemical method in comparison with other techniques usually shows high sensitivity, low cost, and compatibility with microfabrication technology. Good examples of this approach involving dendrimer molecules are gold

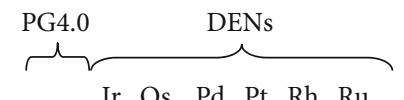

A

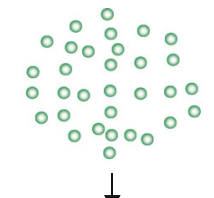

$\downarrow$

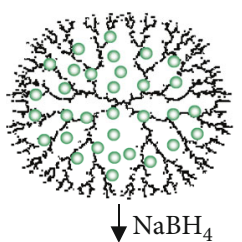

B
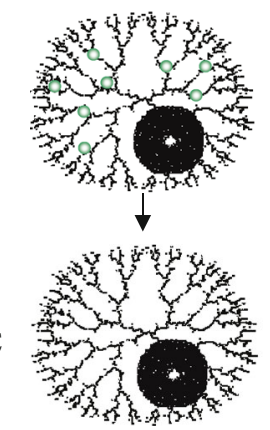

Ir $\mathrm{Os} \mathrm{Pd} \mathrm{Pt} \mathrm{Rh} \mathrm{Ru}$

1

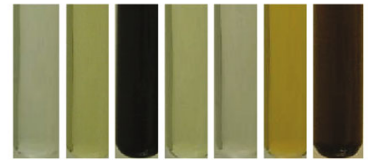

2

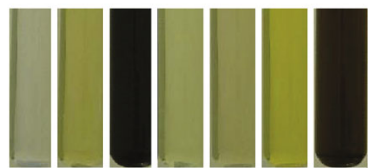

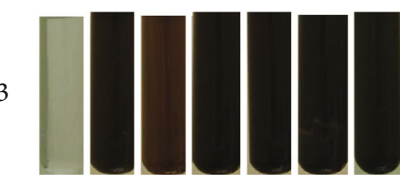

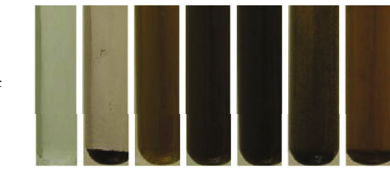

5

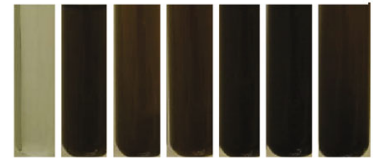

FiguRe 7: Methodology used to prepare dendrimer aggregates(PG4.0-) metal nanoparticles (DENs): (A) dendrimer-ion complexes, (B) ion chemical reduction, and (C) dialysis to purify the DENs formed. Images $1,2,3,4$, and 5 of the solutions prepared in each of the steps of the methodology to obtain nanoparticles: Ir, Os, Pd, Pt, Rh, and Ru.

electrodes modified with ferrocenyl-tethered poly(amido amine) dendrimers linked to carboxylic or aminoterminated self-assembled monolayers (NPs, Figure 6).

5.2. Electrochemical Detection of $\mathrm{H}_{2} \mathrm{O}_{2}$. Another interesting type of sensor that has been studied using dendrimers consists of modified surfaces to detect $\mathrm{H}_{2} \mathrm{O}_{2}$. In this way, cystamine-modified gold substrates were used to attach PAMAM dendrimer generation 4.0 through glutaraldehyde cross-linking. The effect of the dendrimer structure and terminal functional groups in the presence of horseradish peroxidase increased the sensitivity of the system for the detection of $\mathrm{H}_{2} \mathrm{O}_{2}$. 
5.3. Electrochemical Detection of Glutamate. For the electrochemical detection of glutamate, an amperometric biosensor based on the self-assembly of glutamate dehydrogenase (GLDH) and poly(amido amine) dendrimer-encapsulated platinum nanoparticles (Pt-DENs) on multiwalled carbon nanotubes (CNTs). The (GLDH/Pt-DENs)n/CNTs/Ppy hybrid film obtained by electropolymerization of pyrrole on glassy carbon electrodes resulted in a substrate capable of detecting glutamate.

5.4. Electrochemical Detection of Dopamine. Another interesting example is the amperometric detection of dopamine in urine samples using glassy carbon electrodes modified with hydroxyl-terminated PAMAM dendrimer generation 4.0 containing different metallic nanoparticles (Figure 7). The resulting surface-attached nanocomposite was coupled to a high-performance liquid chromatography (HPLC) system, showing good sensing performance and no matrix effects. Similar electrochemical detection has been reported for platinumcontaining dendrimers, Pt-DENs, coupled to a flow injection amperometric (FIA) detector.

\section{Conclusion}

As has been mentioned several times in this work, there are important specific features of dendrimer molecules that make them an interesting class of compounds. The branched structure, internal void regions, and supramolecular internal and external capabilities of these unimolecular covalent micelles make them attractive as drug delivery agents, porous polymeric environments for controlled transport processes, and surface-attached films for biosensor applications.

\section{Conflicts of Interest}

The authors declare that there is no conflict of interest regarding the publication of this paper.

\section{Acknowledgments}

The authors would like to thank Consejo Nacional de Ciencia y Tecnología de México (CONACyT), Fondo Mixto- (FOMIX-) Querétaro, and COLCIENCIAS, code 110152128336, contract RC4492011, for the funding of this research. B. Noriega-Luna is grateful to CONACyT for her postdoctoral scholarship. The authors also want to thank J. Dye, US Peace Corps volunteer at CIDETEQ, for his valuable comments and English revision of this manuscript.

\section{References}

[1] B. Noriega-Luna, L. A. Godínez, F. J. Rodríguez et al., “Applications of dendrimers in drug delivery agents, diagnosis, therapy, and detection," Journal of Nanomaterials, vol. 2014, Article ID 507273, 19 pages, 2014.

[2] V. Gajbhiye, V. K. Palanirajan, R. K. Tekade, and N. K. Jain, "Dendrimers as therapeutic agents: a systematic review," Journal of Pharmacy and Pharmacology, vol. 61, no. 8, pp. 9891003, 2009.
[3] J. M. Oliveira, A. J. Salgado, N. Sousa, J. F. Mano, and R. L. Reis, "Dendrimers and derivatives as a potential therapeutic tool in regenerative medicine strategies-a review," Progress in Polymer Science, vol. 35, no. 9, pp. 1163-1194, 2010.

[4] D. Astruc, E. Boisselier, and C. Ornelas, "Dendrimers designed for functions: from physical, photophysical, and supramolecular properties to applications in sensing, catalysis, molecular electronics, photonics, and nanomedicine," Chemical Reviews, vol. 110, no. 4, pp. 1857-1959, 2010.

[5] S. Svenson, "Dendrimers," in Kirk-Othmer Encyclopedia of Chemical Technology, vol. 26, pp. 786-812, Copyright John Wiley \& Sons, Inc, 2006.

[6] N. Joshi and M. Grinstaff, "Applications of dendrimers in tissue engineering," Current Topics in Medicinal Chemistry, vol. 8, no. 14, pp. 1225-1236, 2008. 